\title{
Facile Synthesis of Magnetic Macroporous Polymer/MOF Composites as Separable Catalysts
}

\author{
Lanjun Chen $^{a,{ }^{,}}$, Xiang Ding ${ }^{a, c, *}$, Jia Huo $^{a, b,{ }^{*}}$, Samir El Hankari ${ }^{a}$, and Darren \\ Bradshaw $^{\mathrm{d}, *}$
}

a State Key Laboratory of Chem/Bio-sensing and Chemometrics, College of Chemistry and Chemical Engineering, Hunan University, Changsa, Hunan 410082, P. R. China. E-mail: Jiahuo@hnu.edu.cn.

b Shenzhen Research Institute of Hunan University, Shenzhen 518057, Guangdong, P.R. China

${ }^{c}$ College of Chemistry and Materials Engineering, Hunan University of Arts and Science, Changde 415000, Hunan, P.R. China

${ }^{d}$ School of Chemistry, University of Southampton, Highfield Campus, Southampton SO17 1BJ, UK. E-mail: D.Bradshaw@soton.ac.uk.

${ }^{\#}$ These authors contribute equally to this work.

\begin{abstract}
Design and synthesis of magnetic macroporous metal-organic frameworks (MOFs) have been of significant interest, in order to improve the catalytic efficiency and reusability. In this work, we prepared magnetic macroporous MOF composites based on as-prepared magnetic macroporous polyacrylamides (MMPam). Different MOFs with or without unsaturated coordinative metal sites, including HKUST-1, MOF-2, UiO-66, and Fe-MIL-101(-NH 2$)$ were grown on and within the MMPam matrices. The composites showed hierarchical porosity with both micro- and macropores, which facilitated substrate diffusion to increase the conversion for isomerization of $\alpha$-pinene oxide from $62 \%$ in the case of pure HKUST-1 microparticles to $90 \%$ in the
\end{abstract}


case of HKUST-1@MMPam. The magnetic property also endowed the composites with easy recovery and reasonable reusability.

Keywords: metal-organic frameworks; macroporous polymer; magnetic recyclability; heterogeneous catalysis

\section{Introduction}

Metal-organic frameworks have been considered as one of most attractive multifunctional materials,[1-3] since a combination of porosity with properties such as catalysis, molecular magnetism, conductivity, ferroelectricity and/or luminescence can be easily assembled into one material, which have potential to be used for recyclable catalysis, selective adsorption or separation, ferroelectric capacitor, chemical sensors, light-emitting devices, and, last but not least, biomedicine.[3-7] In general, the multifunctionality in metal-organic frameworks is achieved by incorporating different functional components (organic ligands and metal salts) to form a bulk material.[2, 10-12] For instance, hierarchical porosity[13] is realized by elaborate choice of organic ligands or utilization of supramolecular templates; characteristics of porosity and magnetism[14] are achievable by incorporating paramagnetic metals and/or open-shell organic ligands as magnetic moment carriers; and multi-variate MOFs[2] can be synthesized through incorporating multiple linkers bearing different functional groups. However, building bulk materials with multifunctional properties often encounters some limitations, such as phase separation instead of a single phase because of the different reactivities of functional ligands,[15] extension of porosity to macropores,[12] or molecular magnetism[16] with low Curie temperature due to limited cooperative interactions.

Building a composite of a MOF with other functional materials provides an alternative approach to extend the technological scope for developing multifunctional materials.[17] Fabrication of magnetic MOFs with room 
temperature ferromagnetism is a good example through growth of MOF with magnetic particles, which can be used in the area of magnetic separation or directed drug delivery[18]. So far, three strategies were applied to prepare magnetic MOF composites, including growth of MOFs within magnetic colloids,[19] direct mixing of preformed magnetic particles and MOFs,[20] and repeated layer-by-layer growth of a MOF onto the surfaces of magnetic particles[21]. The former has potential to block the pore of MOFs to influence the guest diffusion within porous frameworks and/or it is difficult to control the amount of incorporated magnetic components; the second strategy needs special interactions between the magnetic particles and MOFs to prevent phase separation and post-treatment with centrifugation and magnetic separation is required to remove excess nanoparticles, and the latter normally requires long fabrication and preparation times. Therefore, it is of great interest to develop a strategy to circumvent the above-mentioned limitations.

In this paper, we demonstrate that a magnetic macroporous polymer can be used as a substrate for growth of MOFs, which avoids embedding magnetic nanoparticles into MOFs, controlling the interaction between magnetic particles and MOFs, or repeated deposition of MOFs, but which also builds macropores to facilitate the diffusion of substrates and products for efficient catalysis. Owing to their high loadings of catalytically active sites within frameworks, MOFs have been widely used in heterogeneous catalysis. But their application in catalysis was often limited by the slow mass diffusion within frameworks because of their inherent microporosity. The diffusion resistance can be reduced through construction of hierarchical pores within MOF materials to decrease the diffusion length. One way is through elaborate choice of organic ligands or utilization of supramolecular or hard templates, but it is often difficult to prepare macroporous MOFs, due to structure interpenetration, complicated fabrication process, or resultant fragile structures.[18] Another approach is using macroporous materials[22-24] as supports for growth of MOFs, which has been performed by Kaskel's[22] and our groups[24] recently who 
demonstrated integrated structured and improved chemical stability of MOFs deposited within macroporous matrices. However, to the best of our knowledge, there are currently no reports that combine both magnetism and macroporous substrates into one system to achieve both rapid mass diffusion and controlled stimuli-sensitive separation efficiency.

\section{Experimental}

\subsection{Chemicals}

All chemicals were purchased from the Sigma Aldrich Chemical Company and used as received without further purification. All organic solvents are reagent grade and used without further purification, and water used in all experiments was dispensed from a Milli-Q Reagent Water System.

\subsection{Synthesis of magnetic macroporous polyacrylamide (MMPam)}

Magnetic macroporous polyacrylamide was prepared using an emulsion-templated method according to reference with minor modification. A typical preparation procedure is as follow: A stock solution of monomers was prepared by mixing acrylamide (AM, 15.33 g), N,N'-methylenebisacrylamide (MBAM, 3.106 g), and poly(vinyl alcohol) (PVA, $10000 \mathrm{~g} / \mathrm{mol}, 80 \%$ hydrolyzed, $2.25 \mathrm{~g}$ ) in $40 \mathrm{ml}$ of distilled water. $4 \mathrm{ml}$ of the stock aqueous solution, $0.4 \mathrm{ml}$ of Triton $\mathrm{X}-405$ and $\mathrm{Fe}_{3} \mathrm{O}_{4}$ nanoparticles (30 nm; 2.5, 5.0 or $10.0 \mathrm{wt} \%)$ were mixed in a vial and stirred using an impeller stirrer. Xylene (6 ml) was added dropwise and APS (200 $\mu 1,10 \mathrm{wt} \%)$ was added during stirring, which was stirred for further $10 \mathrm{~min}$, and then TMEDA

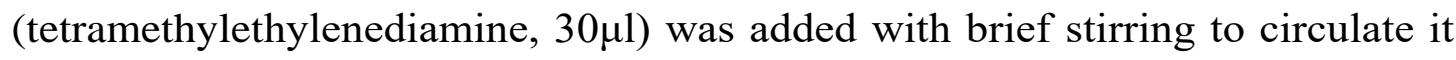
evenly. The resultant emulsion was transferred to a preheated over at $60{ }^{\circ} \mathrm{C}$ for 24 hrs. After removing from the oven, the prepared samples were washed with distilled water and acetone for a few times, cut into small pieces (around $0.3 \mathrm{~cm}$ $\times 0.3 \mathrm{~cm} \times 0.3 \mathrm{~cm}$ ) and then dried under vacuum at $60^{\circ} \mathrm{C}$ overnight. 


\subsection{Synthesis of HKUST-1@MMPam}

HKUST-1@MMPam was prepared through a solvothermal synthesis. A typical procedure to prepare this material is: A stock solution of precursors was prepared by dissolving $\mathrm{Cu}\left(\mathrm{NO}_{3}\right)_{2} \cdot 2.5 \mathrm{H}_{2} \mathrm{O}(2.88 \mathrm{~g})$ and 1,3,5-benzenetricarboxylic acid ( $\mathrm{H}_{3} \mathrm{BTC}, 1.68 \mathrm{~g}$ ) in $10 \mathrm{~g}$ of DMSO. Ten pieces of MMPam were added into mixture of $2 \mathrm{ml}$ of stock solution and $2 \mathrm{ml}$ of methanol in a vial. The vial was sealed and heated to $120{ }^{\circ} \mathrm{C}$ under autogenous pressure for $12 \mathrm{hrs}$. After cooling, the blue composites were separated from precipitated solid with a commercial magnet, washed with fresh $\mathrm{EtOH}$ and dried in an oven at $60{ }^{\circ} \mathrm{C}$.

\subsection{Synthesis of MOF-2@magnetic macroporous polyacrylamide}

Stock solution was prepared by dissolving $\mathrm{Zn}\left(\mathrm{NO}_{3}\right)_{2} .6 \mathrm{H}_{2} \mathrm{O}(1.732$ g, 5.822 mmol) and 1,4-benzenedicarboxylic acid ( $\left.\mathrm{H}_{2} \mathrm{BDC}\right)(0.3217 \mathrm{~g}, 1.938 \mathrm{mmol})$ in $10 \mathrm{~g}$ of DEF $(11 \mathrm{ml})$. To $4 \mathrm{ml}$ of stock solution in a glass sample vial were added 10 magnetic polyacrylamide (PAM) monoliths. The vial was sealed and heated to $105{ }^{\circ} \mathrm{C}$ under autogenous pressure for 24 hours. After cooling, the composite monoliths were separated from precipitated solid using a magnet, washed with fresh $\mathrm{EtOH}$ and dried in an oven at $60{ }^{\circ} \mathrm{C}$ prior to analysis.

\subsection{Synthesis of UiO-66@magnetic macroporous polyacrylamide}

Stock solution was prepared by dissolving $\mathrm{ZrCl}_{4}(0.080 \mathrm{~g}, 0.343 \mathrm{mmol})$, $\mathrm{H}_{2} \mathrm{BDC}(0.057 \mathrm{~g}, 0.343 \mathrm{mmol})$ in $10 \mathrm{ml}$ of DMF. To $2 \mathrm{ml}$ of stock solution in a glass sample vial were added 4 magnetic polyacrylamide (PAM) monoliths. The vial was sealed and heated to $120{ }^{\circ} \mathrm{C}$ under autogenous pressure for 24 hours. After cooling, the composite monoliths were separated from precipitated solid using a magnet, washed with fresh $\mathrm{EtOH}$ and dried in an oven at $60{ }^{\circ} \mathrm{C}$.

\subsection{Synthesis of Fe-MIL-101@magnetic macroporous polyacrylamide}

Stock solution was prepared by dissolving $\mathrm{FeCl}_{3} .6 \mathrm{H}_{2} \mathrm{O}(0.688$ g, 2.545 mmol), $\mathrm{H}_{2} \mathrm{BDC}(0.235 \mathrm{~g}, 1.414 \mathrm{mmol})$ in $15 \mathrm{ml}$ of DMF. Synthetic procedure 
to sample 1: To $4 \mathrm{ml}$ of stock solution containing $\mathrm{FeCl}_{3} \cdot 6 \mathrm{H}_{2} \mathrm{O}$ and $\mathrm{H}_{2} \mathrm{BDC}$ in a glass sample vial were added 10 magnetic polyacrylamide (PAM) monoliths. The vial was sealed and heated to $110{ }^{\circ} \mathrm{C}$ under autogenous pressure for 24 hours. After cooling, the composite monoliths were separated from precipitated solid using a magnet, washed with fresh EtOH and dried in an oven at $60{ }^{\circ} \mathrm{C}$ prior to analysis.

\subsection{Synthesis of Fe-MIL-101- $\mathrm{NH}_{2} @$ magnetic macroporous polyacrylamide}

Stock solution was prepared by dissolving $\mathrm{FeCl}_{3} .6 \mathrm{H}_{2} \mathrm{O}(0.675 \mathrm{~g}, 2.497$ mmol), 2-amino terephthalic acid ( $\left.\mathrm{NH}_{2}-\mathrm{H}_{2} \mathrm{BDC}\right)(0.225 \mathrm{~g}, 1.242 \mathrm{mmol})$ in 15 $\mathrm{ml}$ of DMF. To $4 \mathrm{ml}$ of stock solution in a glass sample vial were added 10 magnetic polyacrylamide (PAM) monoliths. The vial was sealed and heated to $110{ }^{\circ} \mathrm{C}$ under autogenous pressure for 24 hours. After cooling, the composite monoliths were separated from precipitated solid using a magnet, washed with fresh $\mathrm{EtOH}$ and dried in an oven at $60{ }^{\circ} \mathrm{C}$ prior to analysis.

\subsection{Catalytic activity of HKUST-1@MMPam}

Before the reaction, the catalyst samples were dried under vacuum overnight at $100{ }^{\circ} \mathrm{C}$. As for HKUST-1@MMPam, 100 mg of composites (HKUST-1 = 54 wt\%) were dispersed in $2.5 \mathrm{ml}$ of anhydrous ethyl acetate. Then, $54 \mathrm{mg}$ of $\alpha$-pinene oxide and $20 \mu \mathrm{l}$ of mesitylene (internal reference) were added. During the synthesis of composites, pure HKUST-1 prepared in the solution was collected after removing the monoliths for the control experiment. As for heterogeneous catalysis with pure HKUST-1, $54 \mathrm{mg}$ of the HKUST-1 were dispersed in $2.5 \mathrm{ml}$ of anhydrous ethyl acetate. Then, $54 \mathrm{mg}$ of $\alpha$-pinene oxide and $20 \mu \mathrm{L}$ of mesitylene (internal reference) were added. The reactions were carried out at room temperature. The reaction was analysed by ${ }^{1} \mathrm{H}-\mathrm{NMR}$ $\left(\mathrm{CDCl}_{3}\right)$.

\subsection{Instrumental and methods}


Powder X-ray diffraction patterns were collected on a Bruker D2 phaser in the angular range $2 \theta=5-40^{\circ}$ employing a $\mathrm{Ni} \mathrm{K} \beta$ filter (detector side) producing $\mathrm{Cu}\left(\mathrm{K}_{\alpha 1} / \mathrm{K}_{\alpha 2}\right)$ radiation. Thermogravimetric analysis (TGA) was performed using a TG 209 F1 Libra (Netzsch) and the sample was heated from room temperature to $900{ }^{\circ} \mathrm{C}$ at a rate of $10{ }^{\circ} \mathrm{C}$ min-1 under an air atmosphere. Scanning electron microscopy (SEM) measurements were made on a JEOL JSM 6500 thermal field emission scanning electron microscope at an accelerating voltage of 5, 10, or $15 \mathrm{kV}$. Samples for SEM measurements were prepared by firstly placing a drop of capsule suspension in absolute ethanol on a silica wafer attached to an aluminium substrate with a carbon paste that is dried under vacuum overnight, and then sputter-coated with a thin layer of conductive gold to improve electrical conductivity. ${ }^{1} \mathrm{H}-\mathrm{NMR}$ spectra were acquired using a Bruker DPX400 FT-NMR spectrometer.

$\mathrm{N}_{2}$ adsorption/desorption isotherm was measured at $77 \mathrm{~K}$ using a JW-BK200C (Beijing JWGB Sci.\&Tech. Co., Ltd.) gas adsorption device after the sample was first degassed at $100{ }^{\circ} \mathrm{C}$ overnight. Surface areas were determined by the BET method in an appropriate pressure range, and total pore volume was determined using the adsorption branch of $\mathrm{N}_{2}$ isotherm curve at the $\mathrm{p} / \mathrm{p}^{0}=0.99$ single point. Pore size distribution was determined using the adsorption branch of $\mathrm{N}_{2}$ isotherms. Micropore size distribution analysis was carried out using the Horvath-Kawazoe method. Mesopore size distribution was calculated using the Barrett-Joyner-Halenda (BJH) method.

\section{Results and Discussion}

\subsection{Synthesis and characterization of magnetic macroporous polyacrylamide}

Macroporous polyacrylamide[24,25] was selected as a polymer matrix owing to the terminal amide functionality distributed throughout the structures as a potential anchoring point to be used for the further growth of MOFs. Magnetic 
macroporous polyacrylamide (shortened as MMPam) was prepared using an emulsion-templated oil/water (o/w) polymerization technique from a mixture of $\mathrm{Fe}_{3} \mathrm{O}_{4}$ nanoparticles, acrylamide and $\mathrm{N}, \mathrm{N}^{\prime}$-methylenebisacrylamide (see details in Scheme 1 and experimental section).

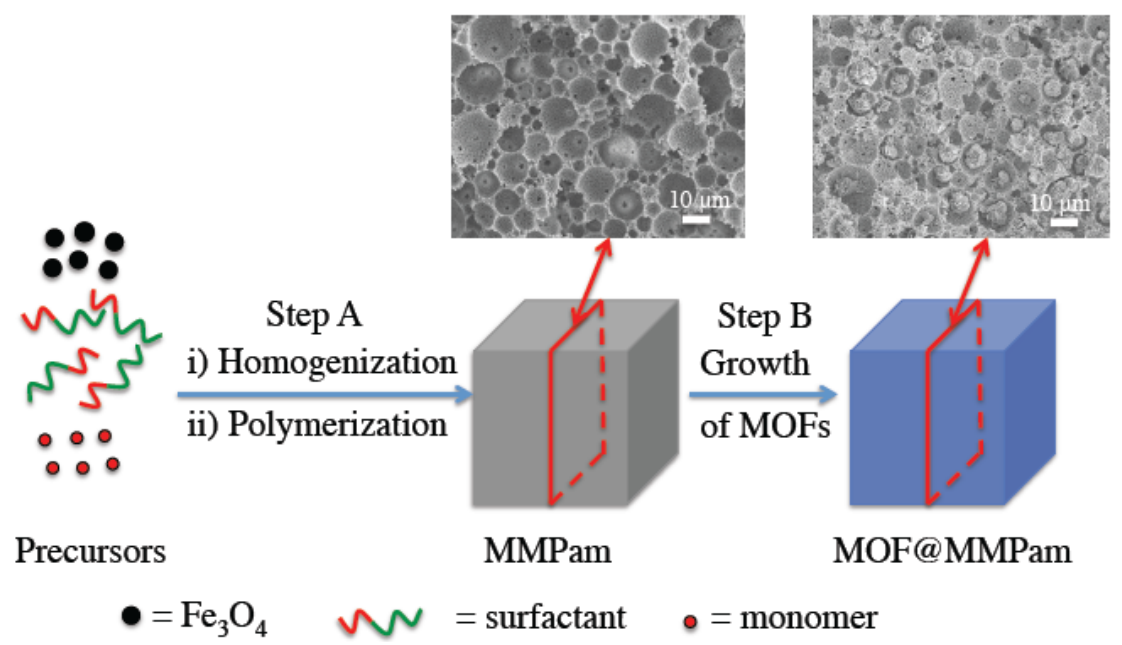

Scheme 1 Synthetic routes for the synthesis of MMPam and MOF@MMPam.

The materials were characterized by SEM, EDX, XRD, TGA, and $\mathrm{N}_{2}$ adsorption/desorption. SEM images of the MMPam matrices indicate that the materials have macropores in the range of 5-15 $\mu$ m (Figure 1). The $\mathrm{Fe}_{3} \mathrm{O}_{4}$ nanoparticles within the MMPam were not able to be observed directly from the SEM images, most likely as a result of being embedded within the polymer. Bragg reflections at 30,35 and $40^{\circ} 2 \theta$ are however observed in the PXRD pattern of the MMPam composite, which are readily assigned to the position of (220), (311), (400), (422), and (511), and match very well with those of bare $\mathrm{Fe}_{3} \mathrm{O}_{4}$ nanoparticles [26]. These reflections can be indexed to the face-centered cubic (fcc) magnetite (JCPDS No.65-3107) lattice, confirming the successful incorporation of magnetic particles within the as-made macroporous polyacrylamides. The broad peak centred at $2 \theta=15^{\circ}$ arises from the amorphous nature of the MMPam matrix. The successful incorporation of magnetic particles was further confirmed by the presence of Fe and $\mathrm{O}$ in the EDX pattern and element mapping (Figure $1 \mathrm{a}$ and $1 \mathrm{c}$ ), which confirm that the $\mathrm{Fe}_{3} \mathrm{O}_{4}$ 
nanoparticles are distributed homogeneously within the porous polymer network.

This single-step approach through direct mixing of $\mathrm{Fe}_{3} \mathrm{O}_{4}$ nanoparticles with monomers before polymerization, where the nanoparticles serve as a co-stabilizer along with Triton X-405 (Figure 1), can produce stable magnetic macroporous polymers that do not collapse after removing solvents and surfactants. We also tried a two-step method to prepare magnetic macroporous polymers by soaking pre-formed polymer matrices in and aqueous dispersion of $\mathrm{Fe}_{3} \mathrm{O}_{4}$ nanoparticles aqueous; however, the resulting structure easily collapsed after removing solvents because of capillary shrinkage. Through this single-step method, the contents of the magnetic particles within the MMPam can be easily and precisely controlled by adjusting the ratio between $\mathrm{Fe}_{3} \mathrm{O}_{4}$ nanoparticles and monomers. TGA results (Figure S2, S3, and S5) show that the measured amounts of magnetic particles at different starting concentrations match well with the theoretical values $(2.22,4.46,8.42 \mathrm{wt} \%$ for TGA determined values $v s$ 2.5, 5.0, and $10.0 \mathrm{wt} \%$ for the theoretical (i.e. 100\%) inclusion level).
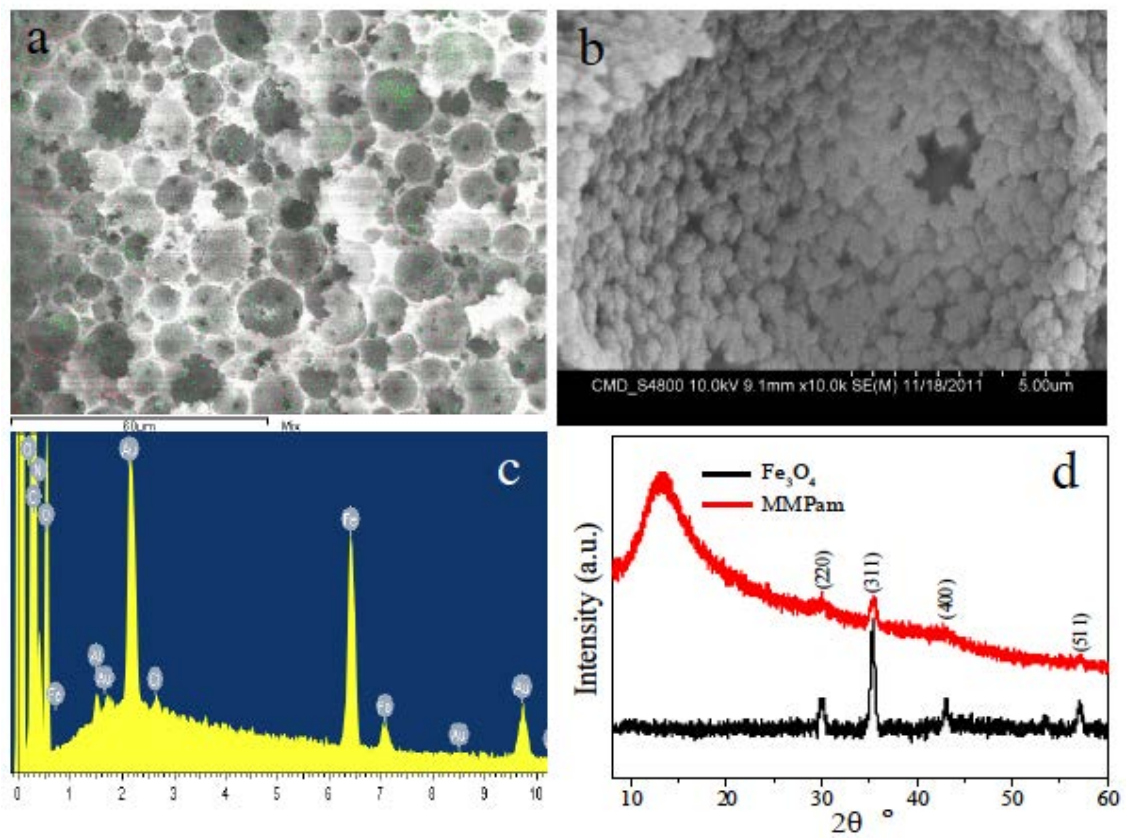

Fig 1. Low (a) and high (b) magnification SEM images of the cross-section of an MMPam monolith (green and red spots in (a) represent the distribution of iron and oxygen, respectively from EDX element mapping), (c) EDX spectrum of MMPam, and (d) PXRD patterns of $\mathrm{Fe}_{3} \mathrm{O}_{4}$ and MMPam. 


\subsection{Synthesis and characterization of HKUST-1@magnetic macroporous polyacrylamide}

We first selected HKUST-1, $\left[\mathrm{Cu}_{3}(\mathrm{BTC})_{2}\right]$ (where BTC = 1,3,5-benzenetricarboxylate)[27] as a model for the preparation of MOFs@MMPam composites, because this prototypical MOF has high loadings of coordinatively unsaturated $\mathrm{Cu}$ sites allowing it to act as a promising acidic heterogeneous catalyst for a range of transformations including: the oxidation of $p$-benzoquinone, cyanosilylation of aldehydes, isomerization of $\alpha$-pinene oxide, cyclization of citronellal, rearrangement of ethylene ketal of 2-bromopropiophenone, and Friedländer reaction.[28-30] The open-metal sites supported by the paddlewheel secondary building units (SBUs) in HKUST-1, also have the potential to interact with the terminal amide of the macroporous polyacrylamide surface. The HKUST-1@magnetic macroporous polyacrylamide (HKUST-1@MMPam) was prepared by soaking the magnetic macroporous polyacrylamide monoliths (containing 4.46 wt\% $\mathrm{Fe}_{3} \mathrm{O}_{4}$ nanoparticles, denoted as MMPam-5) into a MOF precursor solution containing metal salt $\left(\mathrm{Cu}(\mathrm{OAc})_{2}\right)$ and $\mathrm{H}_{3} \mathrm{BTC}$ ligand in a mixture of DMSO/MeOH $(1: 1$, v/v) for 7 days followed by heating at $120{ }^{\circ} \mathrm{C}$ for 12 hrs. Typically HKUST-1 is synthesized within DMF, ethanol, or $\mathrm{H}_{2} \mathrm{O}$, but the products would precipitate rapidly or even immediately under these conditions, which we have demonstrated will restrict the growth of HKUST-1 to the MMPam exterior. (Figure S6). By contrast, using DMSO as the reaction solvent will prevent or slow down the nucleation at room temperature due to the stabilizing effect resulting from favorable interaction with $\mathrm{Cu}^{2+}$ and/or formation of a hydrogen bonded solvate structure with the trimesic acid ligand. Therefore, soaking of monoliths within a solution of precursors without any precipitate will be beneficial for the growth of MOFs within the interior surface of macroporous polymers. 
After the MOF synthesis was completed, the colour of the magnetic monliths changed from grey to blue, indicating that HKUST-1 had been successfully deposited on the surface of the MMPam. The magnetic property of the resultant materials facilitates their separation from any non-incorporated MOF particles and subsequent washing steps using a commercial magnet. The structure of the MOF of the HKUST-1@MMPam-5-C (the composites were named as HKUST-1@MMPam-n-mC, where n and m means the weight percentage of $\mathrm{Fe}_{3} \mathrm{O}_{4}$ and the concentration of reagents relative with that in the experimental section) was confirmed by PXRD as shown in Figure 2, with all observed Bragg reflections indexed to the cubic Fm3m unit cell of HKUST-1, which are in excellent agreement with those of the simulated pattern of HKUST-1.

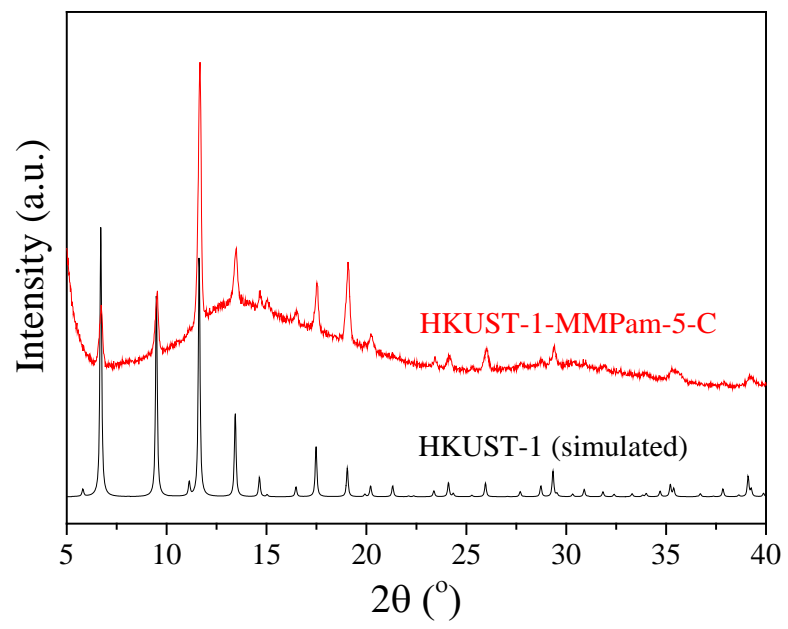

Fig 2. PXRD patterns of HKUST-1@MMPam-5-C composites and HKUST-1 (simulated).

SEM images of the HKUST-1@MMPam-5-C composites show that HKUST-1 was deposited on both the exterior and interior surfaces of the polymer matrix (Figure 3). The HKUST-1 microparticles grown outside show a typical octahedral shape with an average size of $15 \mu \mathrm{m}$. Slow nucleation of MOFs within DMSO/MeOH also allows that smaller octahedral HKUST-1 microparticles are well deposited within the cavities of the macroporous polymers with an average size of $4 \mu \mathrm{m}$, which indicates that the interior of the matrices is fully accessible to the precursor solution and that the macropores may partially limit the growth of HKUST-1 to reduce its crystallite size. By 
contrast, use of $\mathrm{EtOH} / \mathrm{H}_{2} \mathrm{O}$ as solvents under otherwise identical reaction conditions selectively produced HKUST-1 on the exterior surface of the macroporous polymers, possibly because of the rapid nucleation of MOF once precursor solutions were mixed together (Figure S6).

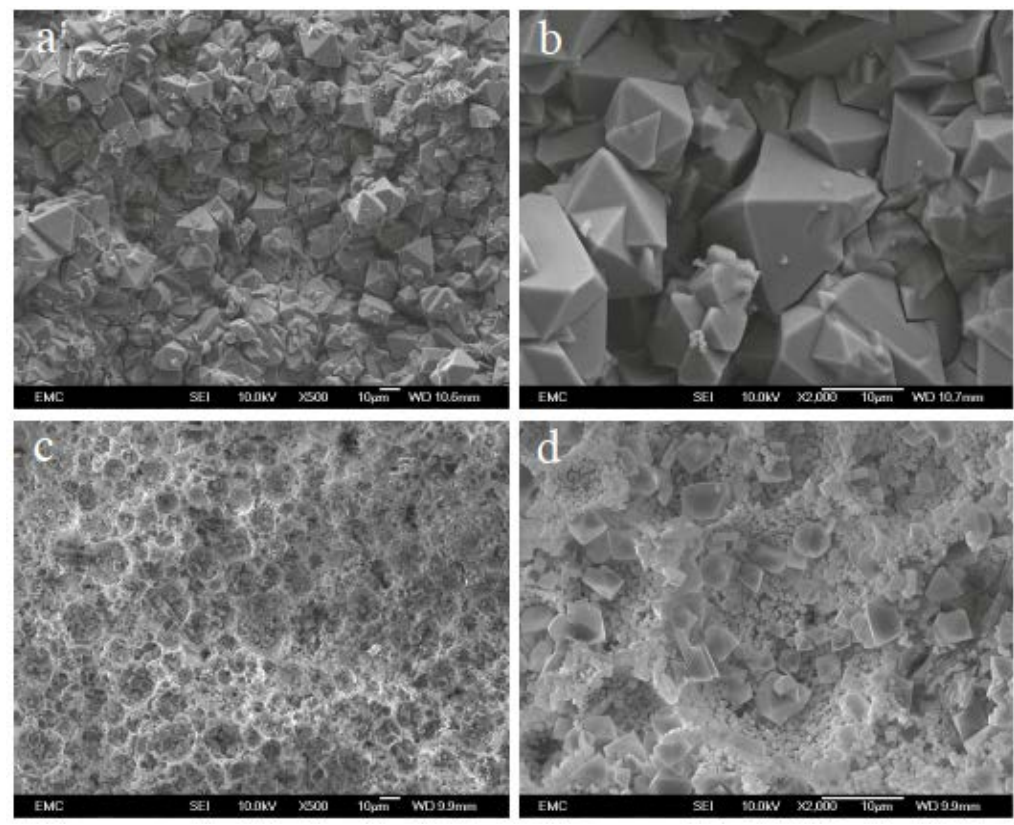

Fig 3. Low (ac) and high (bd) magnification SEM images of exterior (ab) and cross-section (cd) of a HKUST-1@MMPam-5-C monolith.

The quantity of HKUST-1 within the composite was calculated from the TGA data as high as 54.0 wt\% with DMSO/MeOH as solvents, whereas only $35.5 \%$ of HKUST-1 was grown within composites when EtOH/ $\mathrm{H}_{2} \mathrm{O}$ was used as solvents, probably because of limited solubility of precursors and/or selective growth on the exterior. The porosity of the hierarchically porous HKUST-1@MMPam-5-C was studied using $\mathrm{N}_{2}$ sorption isotherms at $77 \mathrm{~K}$ (Figure 4), which revealed a type I isotherm and typical adsorption behavior of a highly microporous system, since any macropores will contribute significantly less to the total $\mathrm{N}_{2}$ uptake. The apparent BET surface area of the composite is $701 \mathrm{~m}^{2} \mathrm{~g}^{-1}$, the total $\mathrm{N}_{2}$ uptake is $200.7 \mathrm{~cm}^{3} \mathrm{~g}^{-1}$ at a relative pressure $\mathrm{p} / \mathrm{p}_{0}=0.99,62 \%$ of that observed for the bulk HKUST-1 $\left(321.4 \mathrm{~cm}^{3}\right.$ $\mathrm{g}^{-1}$ ), and the total pore volume is $0.34 \mathrm{~cm}^{3} \mathrm{~g}^{-1}$. The amount of HKUST-1 within the composites can be controlled by changing the concentration of the precursor solutions. By reducing the concentration by half, the content of HKUST-1 (in 
HKUST-1@MMPam-5-C/2) decreased to 43.7 wt\% and the apparent BET surface area and the total $\mathrm{N}_{2}$ uptake to $626.3 \mathrm{~m}^{2} \mathrm{~g}^{-1}$ and $184.3 \mathrm{~cm}^{3} \mathrm{~g}^{-1}$, respectively. The content of HKUST-1 decreased to $27.9 \mathrm{wt} \%$ and the apparent BET surface area and the total $\mathrm{N}_{2}$ uptake to $515.0 \mathrm{~m}^{2} \mathrm{~g}^{-1}$ and $153.3 \mathrm{~cm}^{3} \mathrm{~g}^{-1}$, respectively, when the concentration was a quarter of the original concentration (HKUST-1@MMPam-5-C/4).

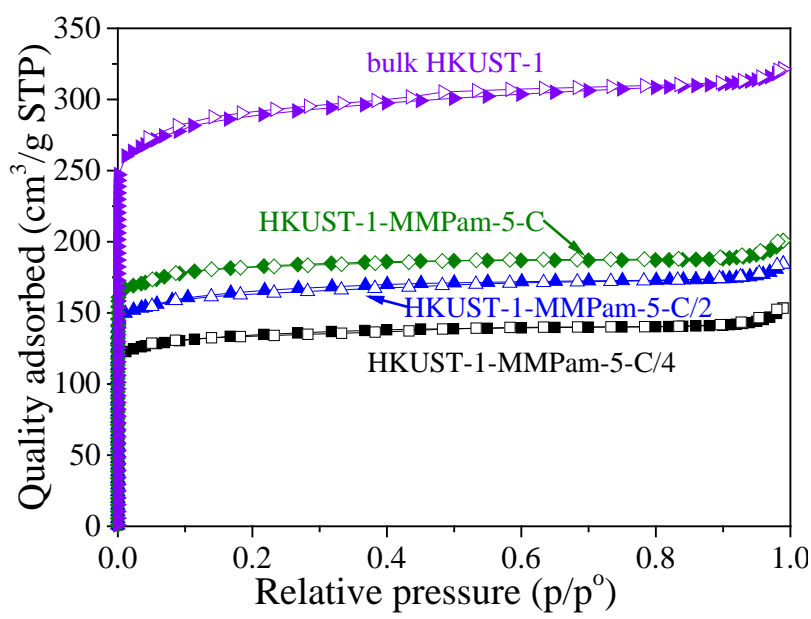

Fig 4. $\mathrm{N}_{2}$ adsorption/desorption isotherms of the bulk HKUST-1 and HKUST-1@MMPam composites measured at $77 \mathrm{~K}$ (closed circles, adsorption; open circles, desorption). HKUST-1@MMPam-5-C is at the original concentration of $0.4 \mathrm{~mol} / \mathrm{l}$ based on $\mathrm{H}_{3} \mathrm{BTC}$ and HKUST-1@MMPam-5-C/2 and HKUST-1@MMPam-5-C/4 are $0.5 \mathrm{x}$ and 0.25 x, respectively.

\subsection{Synthesis and characterization of flower-like MOF-2@magnetic macroporous polyacrylamide}

We have also investigated the growth of MOF-2, [Zn(BDC)], (where BDC = 1,4-benzenedicarboxylate) into magnetic macroporous polyacrylamide. MOF-2 also has paddlewheel SBUs, similar to HKUST-1, but is a two-dimensional carboxylate-derived framework structure.[31] The preparation procedure is similar to that for the synthesis of HKUST-1@MMPam, in which the magnetic macroporous polyacrylamide monoliths (containing $5 \% \mathrm{Fe}_{3} \mathrm{O}_{4}$ nanoparticles) were soaked in a solution containing $\mathrm{Zn}\left(\mathrm{NO}_{3}\right)_{2}$ and the $\mathrm{H}_{2} \mathrm{BDC}$ ligand in DEF for 2 days, which was then heated at $100{ }^{\circ} \mathrm{C}$ for 12 hrs. The structure is confirmed by PXRD (Figure S9) with all observed Bragg reflections indexed to the tetragonal unit cell with P4 symmetry consistent with MOF-2. The content of MOF-2 within the macroporous 
polymer was calculated as $23.8 \mathrm{wt} \%$ from TGA data. The apparent BET surface area and the total $\mathrm{N}_{2}$ uptake of the composite is $67.9 \mathrm{~m}^{2} \mathrm{~g}^{-1}$, and $34.8 \mathrm{~cm}^{3} \mathrm{~g}^{-1}$, respectively.

MOF-2 was grown on both the exterior and interior of the macroporous polymer monolith, as evidenced by the SEM images in Figure 5. Interestingly, instead of formation of block-like MOF particles on the exterior surface, flower-like microparticles were produced with an average length of $5 \mu \mathrm{m}$ within the cavities of the macroporous polymers. The three-dimensional flower-like structures were assembled by a large number of nanosheets with an average thickness of $15 \mathrm{~nm}$. The morphology is similar to that of $\mathrm{ZnO}$ [32] although there is no evidence for the presence of this; therefore, we propose they have a similar formation mechanism, involving the following three steps: (i) nucleation and growth of MOF-2 nanoparticles, (ii) formation of nanosheets through oriented attachment of MOF-2 nanoparticles, and (iii) self-assembly of nanosheets into 3-D flower-like structures. We also observed some separate nanosheets indicating the whole process underwent the step (ii).
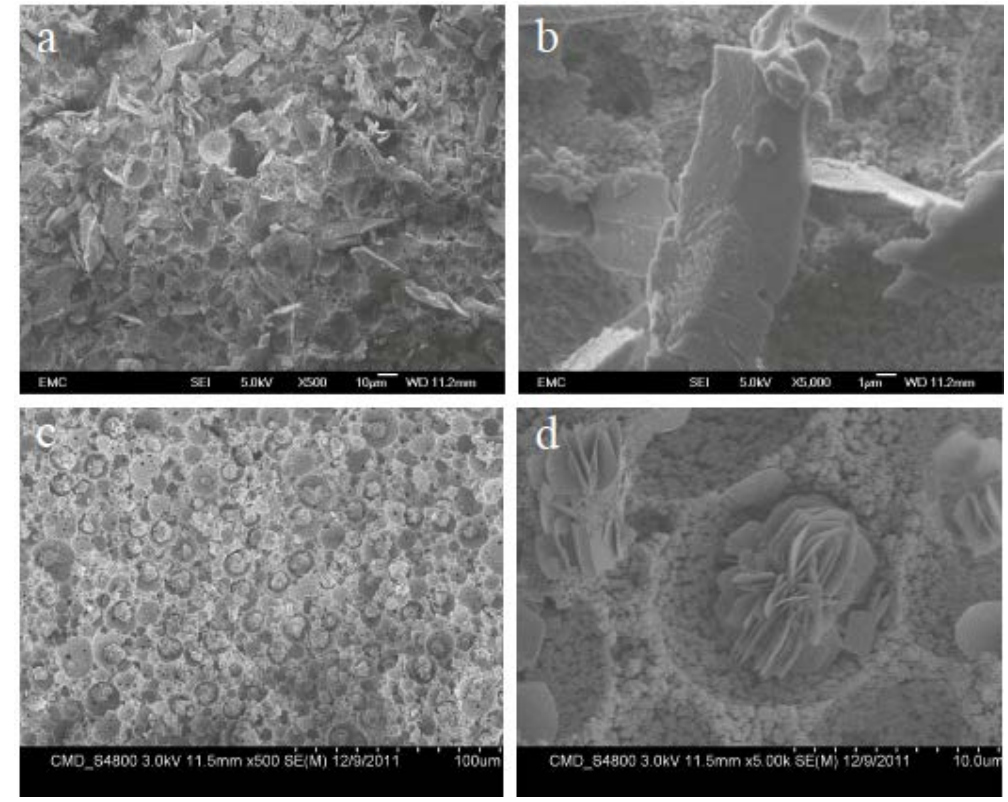

Fig 5. Low (ac) and high (bd) magnification SEM images of exterior (ab) and cross-section (cd) of a MOF-2@MMPam monolith.

\subsection{Synthesis and characterization of UiO-66@magnetic macroporous polyacrylamide}

UiO-66 is a highly packed fcc structure without coordinatively unsaturated metal 
sites, comprised of $\mathrm{Zr}_{6} \mathrm{O}_{4}(\mathrm{OH})_{4}$ octahedra that are 12-fold connected to adjacent octahedra through a BDC linker ( $\mathrm{UiO}=$ University of Oslo).[33] The synthetic method of UiO-66@MMPam is the same as the above-mentioned ones by soaking magnetic polyacrylamide monoliths within the precursor solutions containing $\mathrm{ZrCl}_{4}$ and $\mathrm{H}_{2} \mathrm{BDC}$ in DMF followed by heating at $120^{\circ} \mathrm{C}$ for 24 hrs. The resultant materials were characterized with PXRD, TGA, $\mathrm{N}_{2}$ isotherm, and SEM. PXRD data confirms the formation of UiO-66 within the porous polymers, in which all observed Bragg reflections are indexed to the cubic Im3m unit cell of phase pure UiO-66 (Fig S14). The TGA profile shows that $17.6 \mathrm{wt} \%$ of UiO-66 was incorporated into the macroporous polymer matrix. The apparent BET surface area of the composite is $202.0 \mathrm{~m}^{2} \mathrm{~g}^{-1}$. SEM images show the presence of UiO-66 nanoparticles with an average diameter of $30 \mathrm{~nm}$ around the wall of the internal macropores (Figure 6), whereas, the particle size is around $100 \mathrm{~nm}$ at the exterior surface (Figure S12). EDX clearly demonstrates the presence of Zr within UiO-66@MMPam and a control sample without growth of UiO-66 but with the same procedure for SEM characterization showed the absence of any nanoparticles, confirming that UiO-66 was deposited in the interior of macroporous polymers (Figure 6d).
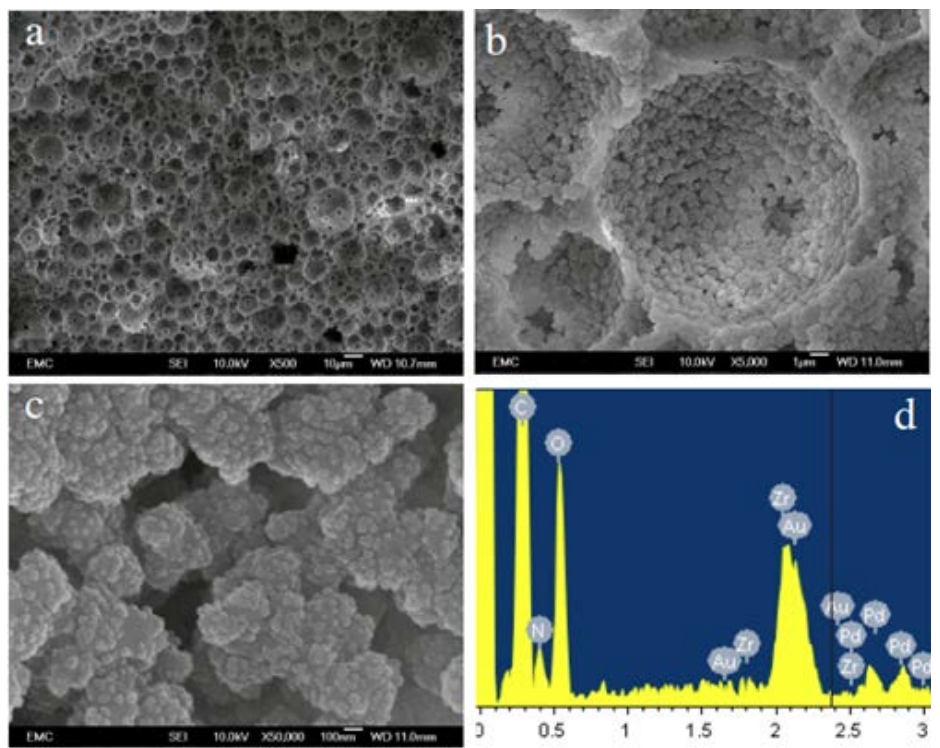

Fig 6 Low (a) and high (bc) magnification SEM images of cross-section of a UiO-66@MMPam monolith, and (d) EDX spectrum of UiO-66@MMPam monolith.

\subsection{Synthesis and characterization of Fe-MIL-101@magnetic macroporous}




\section{polyacrylamide}

Fe-MIL-101[34], $\mathrm{Fe}_{3} \mathrm{OH}\left(\mathrm{H}_{2} \mathrm{O}\right)_{2} \mathrm{O}(\mathrm{BDC})_{3}$, is another MOF incorporated into polymer matrices, which is composed from the linkage of BDC anions with inorganic trimers containing three iron atoms each in an octahedral environment. By soaking magnetic polyacrylamide monoliths within the precursor solution containing $\mathrm{FeCl}_{3}$ and $\mathrm{H}_{2} \mathrm{BDC}$ in DMF for 2 days followed by heating at $120{ }^{\circ} \mathrm{C}$ for 12 hrs, yellow composites were produced. PXRD patterns of the composite (Figure S18) confirms the successful preparation of Fe-MIL-101 with the cubic Fd3m unit cell. TGA analysis showed that there are $17.3 \mathrm{wt} \%$ of MOFs on macroporous polymers. MOF nanoparticles were grown on both interior and mainly exterior surface, as shown by SEM images in Figure 7 and S17. The apparent BET surface area and the total $\mathrm{N}_{2}$ uptake of the composite are $521.7 \mathrm{~m}^{2} \mathrm{~g}^{-1}$, and $237.6 \mathrm{~cm}^{3} \mathrm{~g}^{-1}$, respectively. Fe-MIL-101- $\mathrm{NH}_{2}$ [35], $\mathrm{Fe}_{3} \mathrm{OH}\left(\mathrm{H}_{2} \mathrm{O}\right)_{2} \mathrm{O}\left(\mathrm{NH}_{2}-\mathrm{BDC}\right)_{3}$ was also grown on macroporous polymers under the same procedure. $22.1 \mathrm{wt} \%$ of Fe-MIL-101- $\mathrm{NH}_{2}$ nanoparticles were incorporated into polymer matrices and mostly observed on the interior surface compared with Fe-MIL-101 (Figure 7 and S17). The surface area of the composite is $581.3 \mathrm{~m}^{2} \mathrm{~g}^{-1}$. However, when an isoreticular MIL-101 containing $\mathrm{Al}[36]$ was grown in MMPam matrices, the magnetic separation capability disappeared probably because of the highly acidic conditions during reaction $(\mathrm{pH}=2)$ that likely degraded the incorporated magnetite particles.

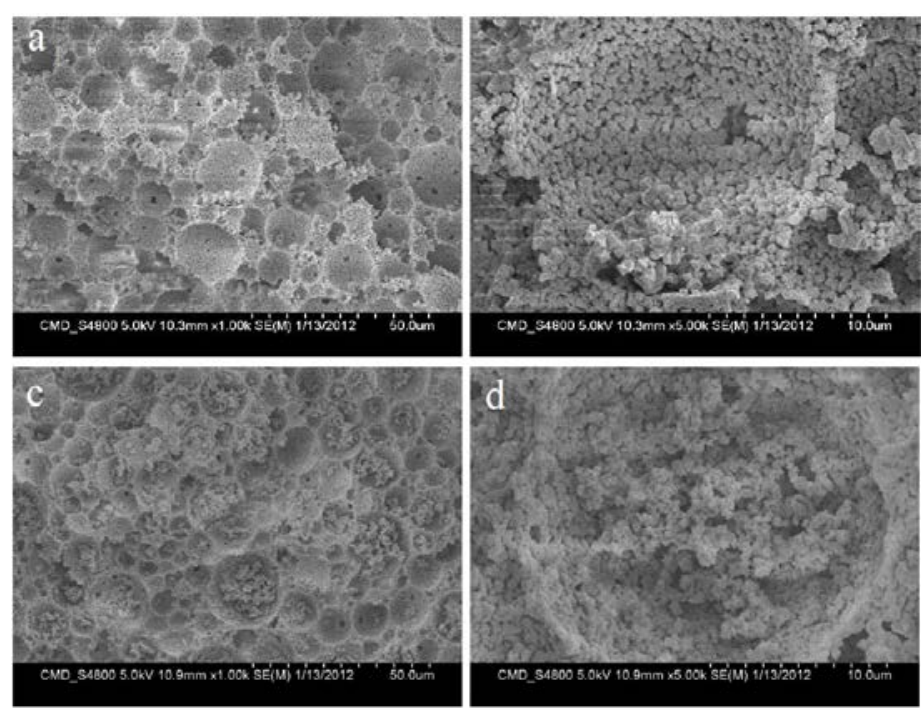

Fig 7. Low (ac) and high (bd) magnification SEM images of Fe-MIL-101@MMPam monolith (ab) 
and Fe-MIL-101-NH2@ MMPam monolith (cd).

\subsection{Catalysis performance of HKUST-1@magnetic macro-porous polyacrylamide}

During the past years, MOFs have been used as heterogeneous catalysts for a wide variety of reactions. In particular, HKUST-1 has been previously used as a Lewis acid catalyst for several important isomerization reactions, such as the conversion of $\alpha$-pinene oxide into campholenic aldehyde (Scheme 2)[30]. The conversion yields and selectivity found by De Vos et al. [30] varies depending on the solvent as well as the synthetic procedure and pre-treatment of the catalyst (including washing and drying treatment). In their report, the highest reaction rate was found in ethyl acetate, with more than $90 \%$ of conversion after 80 h. The selectivity remained constant above $80 \%$ throughout the reaction.

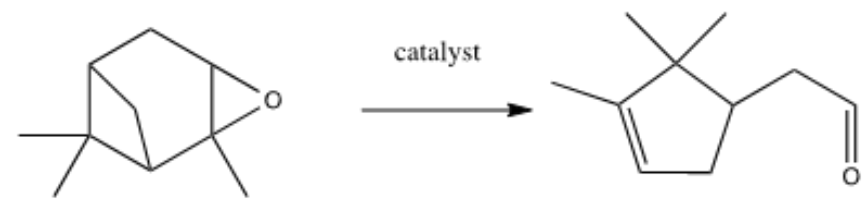

Scheme 2. Isomerization of $\alpha$-pinene oxide to campholenic aldehyde.

Therefore, we selected magnetic macroporous composite with HKUST-1 to evaluate the catalytic activity and the reusability for the conversion of $\alpha$-pinene oxide into campholenic aldehyde. The reaction was carried out under the same conditions previously reported by De Vos and co-workers[30] (see experimental section for details) taking into account the amount of HKUST-1 in the composite, and we compared these results with a pure bulk sample of the HKUST-1 framework. As a control, the catalysis in the presence of the magnetic macroporous polyacrylamide only was also performed. Figure 8 shows the relationship between conversion and reaction time for the isomerization reaction shown in scheme 2. For bulk HKUST-1 (Figure S24), the conversion reached $62 \%$, with selectivity above $63 \%$. Surprisingly, for the composite HKUST-1@MMPam, the conversion after 78 h was as high as $90 \%$, with selectivity of slightly higher than $70 \%$. Since no conversion at all was 
observed for the MMPam itself, we attribute this increase in the conversion and selectivity to the macroporous net of the composite, which can facilitate the diffusion of substrates and products.

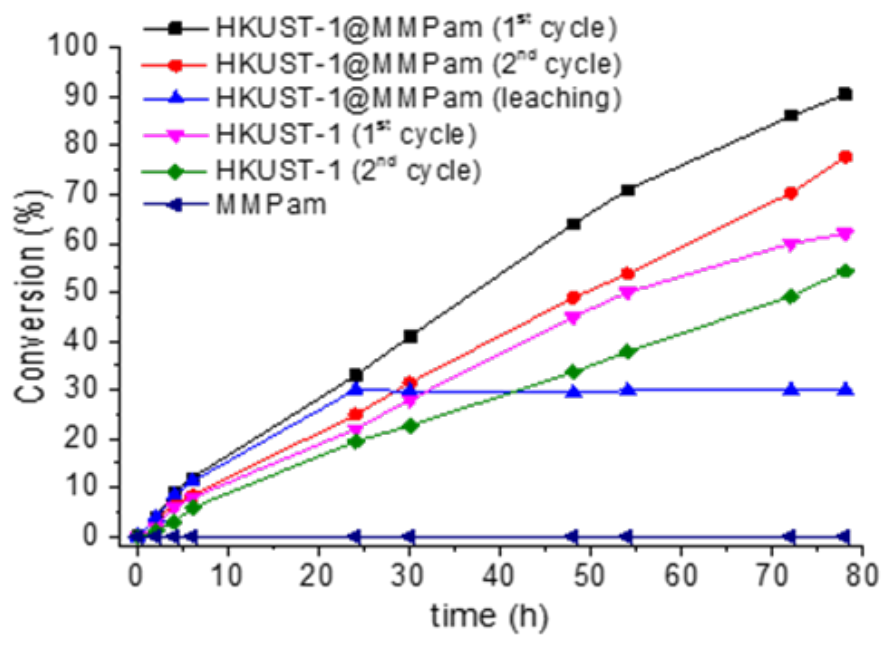

Fig 8. Conversion of $\alpha$-pinene oxide to campholenic aldehyde by using different catalysts.

As described above, the easy-recovery of the composite by a magnet (Figure S25) encouraged us to evaluate its reusability. The samples were washed several times with ethyl acetate or mixture ethanol/water, and dried at $80{ }^{\circ} \mathrm{C}$ overnight. After structural integrity was verified by PXRD (Figure 9) indicating that the composite remained intact in both washing procedures, we carried out the recyclability tests in ethyl acetate. Although the selectivity remains constant after the 2nd cycle, the conversion decreases by around $15 \%$ for both HKUST-1 and the magnetic composite. This decrease, also observed by De Vos and colleagues [30], was attributed to a small loss of catalyst during the washing procedures, which was confirmed by the TGA data (Figure S26). To confirm this, a leaching experiment was also carried out. As shown in Figure 8, after $24 \mathrm{~h}$ of reaction the composite was removed from the mixture, however ${ }^{1} \mathrm{H}$-NMR spectroscopy reveals no further conversion to campholenic aldehyde, although a slightly blue colour in the supernatants can be observed, presumably because HKUST-1 particles become partially detached from the exterior surface of the HKUST-1@MMPam although this is insufficient to be able to continue to catalyze the reaction to a measurable degree. 


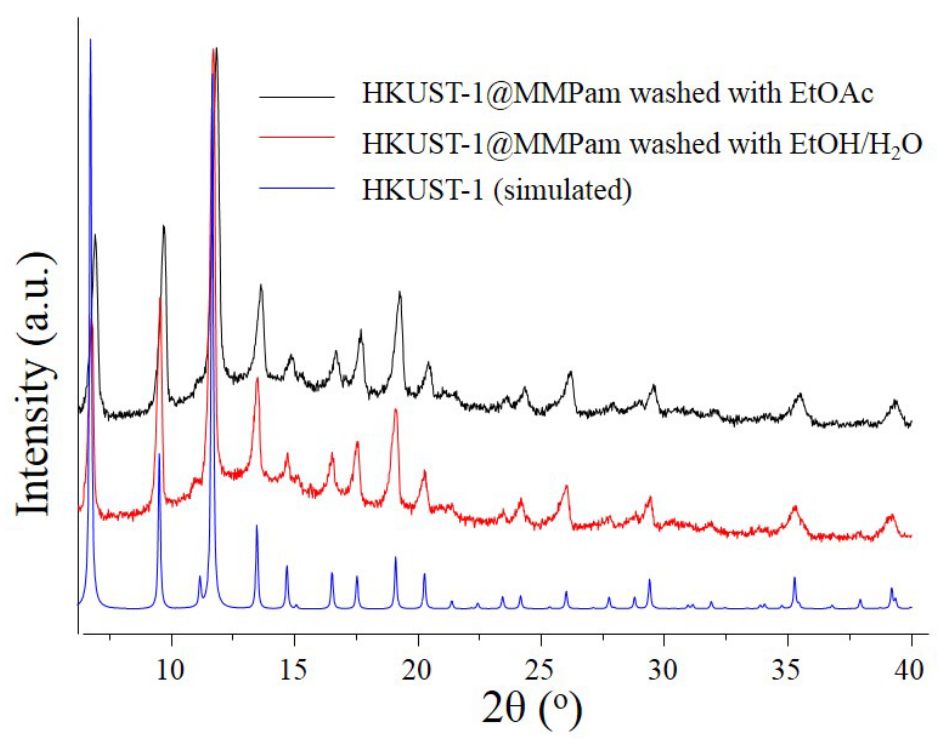

Fig 9. PXRD patterns of the composite washed with EtOAc or EtOH/ $\mathrm{H}_{2} \mathrm{O}$ mixuture after 1st cycle of reaction.

\section{Conclusions}

In conclusion, we have presented a combination approach for the synthesis of magnetic macroporous MOF-polymer composites based on magnetic macroporous polymers to achieve both lower diffusion resistance and recyclability. Various series of MOFs, including HKUST-1, MOF-2, UiO-66, and Fe-MIL-101(-NH 2 ) were grown on macroporous matrices through in-situ growth, which indicated that different types of metal-based building blocks can be grown within the polymer macropores. The MOF particles were grown on both the exterior and interior surfaces and the macropores also influenced the particle morphology from sheets to flower-like particles in the case of MOF-2, and the particle size in the case of other MOFs. The catalytic properties of isomerization of $\alpha$-pinene oxide and recyclability were evaluated for HKUST-1@MMPam, which showed higher conversion and selectivity compared with bulk HKUST-1 particles and enhanced recyclability. The successful combination of MOFs with magnetic macroporous matrices without any effect of MOF itself would provide a promising candidate as a highly efficient catalyst with magnetic recyclability 


\section{Acknowledgements}

The authors acknowledge support from the National Natural Science Foundation of China (Grant No.: 51503062 and 21573063), the European Research Council (ERC-StG-2010-258613-BIOMOF), the Provincial Natural Science Foundation of Hunan (Grant No.: 2017JJ3025), Shenzhen Science and Technology Innovation Committee (Grant No.: JCYJ20170306141630229), and the Fundamental Research Funds for the Central Universities.

\section{References:}

[1] H. Furukawa, K.E. Cordova, M. O’Keeffe, O.M. Yaghi (2013) The Chemistry and applications of metal-organic frameworks, Science, 341: 974.

[2] H. Deng, C.J. Doonan, H. Furukawa, R.B. Ferreira, J. Towne, C.B. Knobler, B. Wang, O.M. Yaghi (2010) Multiple functional groups of varying ratios in metal-organic frameworks, Science, 327: 846-850.

[3] T.R. Zhan, S.S. Lu, H.Q. Rong, W.G. Hou, H.N. Teng, Y.H. Wen (2018) Metal-organic-framework-derived Co/nitrogen-doped porous carbon composite as an effective oxygen reduction electrocatalyst, J. Mater. Sci., 53: 6774-6784.

[4] L.E. Kreno, K. Leong, O.K. Farha, M. Allendorf, R.P. Van Duyne, J.T. Hupp (2011) Metalorganic framework materials as chemical sensors, Chem. Rev., 112: 1105-1125.

[5] P. Horcajada, R. Gref, T. Baati, P.K. Allan, G. Maurin, P. Couvreur, G. Ferey, R.E. Morris, C. Serre (2012) Metal-organic frameworks in biomedicine, Chem. Rev., 112: 1232-1268.

[6] A.C. Tella, S.O. Owalude (2014) A green route approach to the synthesis of Ni(II) and Zn(II) templated metal-organic frameworks, J. Mater. Sci., 49 5635-5639.

[7] J. de Decker, J. de Clercq, P. Vermeir, P. van der Voort (2016) Functionalized metal-organic-framework CMPO@MIL-101(Cr) as a stable and selective rare earth adsorbent, J. Mater. Sci., 51: 5019-5026.

[8] H. Liu, C. Xu, D. Li, H. L. Jiang (2018) Photocatalytic hydrogen production coupled with selective benzylamine oxidation over MOF composites, Angew. Chem., 57: 5379-5383.

[9] Rui Li, Sikai Wu, Xueying Wan, Hangxun Xu, Yujie Xiong (2016) $\mathrm{Cu} / \mathrm{TiO}_{2}$ octahedral-shell photocatalysts derived from metal-organic framework@semiconductor hybrid structures, Inorg. Chem. Front., 3: 104-110.

[10] Ran Long, Yu Li, Yan Liu, Shuangming Chen, Xusheng Zheng, Chao Gao, Chaohua He, Nanshan Chen, Zeming Qi, Li Song , Jun Jiang, Junfa Zhu, and Yujie Xiong (2017) Isolation of $\mathrm{Cu}$ atoms in Pd lattice: Forming highly selective sites for photocatalytic conversion of $\mathrm{CO}_{2}$ to $\mathrm{CH}_{4}$, J. Am. Chem. Soc., 139: 4486-4492.

[11] D. C. Zhong, Y. Q. Wen, J. H. Deng, X. Z. Luo, Y. N. Gong, T. B. Lu (2015) Uncovering the role of metal catalysis in tetrazole formation by an in situ cycloaddition reaction: An experimental approach, Angew. Chem., 54: 11795-11799.

[12] L. M. Cao, J. W. Wang, D. C. Zhong, T. B. Lu (2018) Template-directed synthesis of sulphur doped NiCoFe layered double hydroxide porous nanosheets with enhanced electrocatalytic 
activity for the oxygen evolution reaction, J. Mater. Chem. A, 2018, 6, 3224-3230

[8] D. Bradshaw, S. El-Hankari, L. Lupica-Spagnolo (2014), Supramolecular templating of hierarchically porous metal-organic frameworks, Chem. Soc. Rev., 43: 5431-5443.

[9] M. Kurmoo (2009) Magnetic metal-organic frameworks, Chem. Soc. Rev., 38: 1353-1379.

[10] K. Koh, A.G. Wong-Foy, A.J. Matzger (2008) A crystalline mesoporous coordination copolymer with high microporosity, Angew. Chem. Int. Ed., 47: 677-680.

[11] E. Coronado, M. Giménez-Marqués, G.M. Espallargas, L. Brammer (2012) Tuning the magneto-structural properties of non-porous coordination polymers by $\mathrm{HCl}$ chemisorption, Nat. Commun., 3: 828.

[12] R. Ricco, L. Malfatti, M. Takahashi, A.J. Hill, P. Falcaro (2013) Applications of magnetic metal-organic framework composites, J. Mater. Chem. A, 1: 13033-13045.

[13] I. Imaz, J. Hernando, D. Ruiz-Molina, D. Maspoch (2009) Metal-organic spheres as functional systems for guest encapsulation, Angew. Chem. Int. Ed., 48: 2325-2329.

[14] P. Falcaro, F. Normandin, M. Takahashi, P. Scopece, H. Amenitsch, S. Costacurta, C.M. Doherty, J.S. Laird, M.D.H. Lay, F. Lisi, A.J. Hill, D. Buso (2011) Dynamic control of MOF-5 crystal positioning using a magnetic field, Adv. Mater., 3901-3906.

[15] Y. Hu, Z. Huang, J. Liao, G. Li (2013) Chemical bonding approach for fabrication of hybrid magnetic metal-organic framework-5: High efficient adsorbents for magnetic enrichment of trace analytes, Anal. Chem., 85: 6885-6893.

[16] M.E. Silvestre, M. Franzreb, P.G. Weidler, O. Shekhah, C. Wöll (2013) Magnetic cores with porous coatings: Growth of metal-organic frameworks on particles using liquid phase epitaxy, Adv. Funct. Mater., 23: 1210-1213.

[22] M.G. Schwab, I. Senkovska, M. Rose, M. Koch, J. Pahnke, G. Jonschker, S. Kaskel (2008) MOF@PolyHIPEs, Adv. Eng. Mater., 10: 1151-1155.

[23] L.D. O'Neill, H. Zhang, D. Bradshaw (2010) Macro-/microporous MOF composite beads, J. Mater. Chem., 20: 5720-5726.

[24] G. Li, Q. Liu, B. Xia, J. Huang, S. Li, Y. Guan, H. Zhou, B. Liao, Z. Zhou, B. Liu (2017) Synthesis of stable metal-containing porous organic polymers for gas storage, Eur. Polym. J., 91: 242-247.

[19] L. Qian, A. Ahmed, H. Zhang (2011) Formation of organic nanoparticles by solvent evaporation within porous polymeric materials, Chem. Commmun., 47:10001-10003.

[20] H. Zhang, A.I. Cooper (2007) Aligned porous structures by directional freezing, Adv. Mater., 19: 1529-1533.

[21] Y. Zeng, R. Hao, B. Xing, Y. Hou, Z. Xu (2010) One-pot synthesis of $\mathrm{Fe}_{3} \mathrm{O}_{4}$ nanoprisms with controlled electrochemical properties, Chem. Commun., 46: 3920-3922.

[22] S.S.-Y. Chui, S.M.-F. Lo, J.P.H. Charmant, A.G. Orpen, I.D. Williams (1999) A Chemically Functionalizable Nanoporous Material $\left[\mathrm{Cu}_{3}(\mathrm{TMA})_{2}\left(\mathrm{H}_{2} \mathrm{O}\right)_{3}\right]_{\mathrm{n}}$, Science, 283: 1148-1150.

[23] E. Pérez-Mayoral, J. Čejka (2011) [ $\left.\mathrm{Cu}_{3}(\mathrm{BTC})_{2}\right]$ : A Metal-organic framework catalyst for the Friedländer reaction, Chemcatchem, 3: 157-159.

[24] K. Schlichte, T. Kratzke, S. Kaskel (2004) Improved synthesis, thermal stability and catalytic properties of the metal-organic framework compound $\mathrm{Cu}_{3}(\mathrm{BTC})_{2}$, Microporous Mesoporous Mater., 73: 81-88.

[25] L. Alaerts, E. Seguin, H. Poelman, F. Thibault-Starzyk, P.A. Jacobs, D.E. De Vos (2006) Probing the Lewis acidity and catalytic activity of the metal-organic framework $\mathrm{Cu}_{3}(\mathrm{btc})_{2}$ 
(BTC = benzene-1,3,5-tricarboxylate), Chem. Eur. J., 12: 7353-7363.

[26] H.K. Arslan, O. Shekhah, D.C.F. Wieland, M. Paulus, C. Sternemann, M.A. Schroer, S. Tiemeyer, M. Tolan, R.A. Fischer, C. Wöll (2011) Intercalation in layered metal-organic frameworks: Reversible inclusion of an extended $\pi$-system, J. Am. Chem. Soc., 133: 8158-8161.

[27] Y. Sun, L. Wang, X. Yu, K. Chen (2012) Facile synthesis of flower-like 3D ZnO superstructures via solution route, CrystEngComm, 14: 3199-3204.

[28] J.H. Cavka, S. Jakobsen, U. Olsbye, N. Guillou, C. Lamberti, S. Bordiga, K.P. Lillerud (2008) A new zirconium inorganic building brick forming metal organic frameworks with exceptional stability, J. Am. Chem. Soc., 130: 13850-13851.

[29] N.V. Maksimchuk, K.A. Kovalenko, V.P. Fedin, O.A. Kholdeeva (2012) Cyclohexane selective oxidation over metal-organic frameworks of MIL-101 family: superior catalytic activity and selectivity, Chem. Commun., 48: 6812-6814.

[30] M. Savonnet, E. Kockrick, A. Camarata, D. Bazer-Bachi, N. Bats, V. Lecocq, C. Pinel, D. Farrusseng (2011) Combinatorial synthesis of metal-organic frameworks libraries by click-chemistry, New J. Chem., 35: 1892-1897.

[31] P. Serra-Crespo, E.V. Ramos-Fernandez, J. Gascon, F. Kapteijn (2011) Synthesis and characterization of an amino functionalized MIL-101(Al): Separation and catalytic properties, Chem. Mater., 23: 2565-2572. 